doi: http://dx.doi.org/10.7124/bc.0009D8

\section{C-1. Pericentromeric tandem repeat DNA transcription in mesenchymal stem cells from multiple myeloma patients}

V. Chubar ${ }^{1,2}$, N. U. Semenova ${ }^{4}$, V. I. Rugal ${ }^{4}$, A. V. Kotova ${ }^{1,3,5}$, N. I. Enukashvili1 1,5

${ }^{1}$ Institute of Cytology, Russian Academy of Sciences, St. Petersburg, Russia; ${ }^{2}$ Saint-Peterburg State University, St-Petersburg, Russia; ${ }^{3}$ Pokrovsky Stem Cell Bank, LLC, St. Petersburg, Russia; 4 "Russian Scientific Research Institute of Hematology and Transfusiology FMBA of Russia”, St. Petersburg, Russia; ${ }^{5}$ North-Western State Medical University named after I.I. Mechnikov, St. Petersburg, Russia. nie@newmail.ru

Satellite DNA repeats are tandem arrays located in the centromeric and pericentromeric regions of chromosomes. Tandem repeats (TR) remain silent under normal conditions, but appears to be extensively transcribed in tumor biopsy samples (Ting et al., 2011). In our laboratory, transcription of the pericentromeric TR (human satellite 3, HS3) in solid tumor's microenvironment fibroblasts was shown. The study was aimed on the investigation of HS3 transcripts in mesenchymal stem cells (MSCs) obtained from multiple myeloma (MM) patients with different percentage of plasmatic cells (PC). MSCs are an important cell type forming bone marrow microenvironment that can display genomic alterations in MM which is liquid tumor and has no known cause to date. MSCs were obtained from bone marrow aspirates of 5 patients with $\mathrm{MM}$ and one healthy donor. Median age of MM patients was 59 years, ranging from 49 to 71 years. Two patients had high degree of bone marrow infiltration (from $14 \%$ to $47 \% \mathrm{PC}$ in biopsy) and 3 patients had low (under $5 \%$ PC). The fluorescence in situ hybridization for HS3 was performed on slides with fixed cells. In MSCs, the amount of HS3 transcripts correlated with the severity of the disease. All signals were localized in nucleus and not in cytoplasm and disappeared after RNase treatment. MSCs from patients with high percentage of plasma cells displayed higher HS3 transcriptional activity than ones with low infiltration. Two of three patients with single tumor cells were negative for HS3 while the third one had no treatment and exhibited MSCs positive for this satellite. MSCs from healthy donors displayed no HS3 transcriptional activity. HS3 transcription in MSCs from MM correlated with cancer-associated phenotype that included smooth muscle actin and SA- $\beta$-gal expression. In conclusion, HS3 transcription in bone marrow microenvironment is associated with the progression of MM and can indicate a poorer prognosis.

The work was supported by grants from the Grants' Council of the President of RF (MK-6706.2018.7) and RSF (19-74-20102).

References: Ting, D. T., Lipson, D., Paul, S., Brannigan, B. W., Akhavanfard, S., Coffman, E. J., et al. (2011). Aberrant overexpression of satellite repeats in pancreatic and other epithelial cancers. Science, 331(6017), 593-596

doi: http://dx.doi.org/10.7124/bc.0009D9

\section{D-1. Nuclear function of vinculin in mouse primary oocytes}

Alžběta Darášová ${ }^{1}$, Petr Flachs ${ }^{1}$, Robert Havalda $^{1}$, Pavel Hozák ${ }^{1,2}$

${ }^{1}$ Laboratory of Epigenetics of the Cell Nucleus, Institute of Molecular Genetics AS CR, division BIO$\mathrm{CEV}$, Vestec, Czech Republic; ${ }^{2}$ Laboratory of the 